\title{
Development of the Real-Time Subjective Emotionality Assessment (RTSEA) system
}

\author{
Soon-Cheol Chung \\ Konkuk University, Chungju, Korea \\ Yoon-KI Min \\ Chungnam National University, Daejeon, Korea \\ Bongsoo Lee, Gye-Rae Tack, Jeong-Han Yi, Ji-Hye You, and Jae-Hun Jun \\ Konkuk University, Chungju, Korea \\ AND \\ Byung-Chan Min \\ Hanbat National University, Daejeon, Korea

\begin{abstract}
A new Real-Time Subjective Emotionality Assessment (RTSEA) system was developed for this study. The system is composed of two parts: an emotionality input and evaluation parts. An experiment was conducted in order to investigate the effectiveness of the RTSEA system. The present study compared Galvanic Skin Response (GSR) with the RTSEA by presenting 28 subjects with pictures that aroused either positive or negative emotion. Following the experiment, a subjective assessment using a questionnaire was given to the same subjects. According to the correlation coefficients, changes of the RTSEA had strong correlations with the changes of the GSR. Also, the questionnaire results showed marked similarity to the average responses of the RTSEA. In conclusion, the most remarkable characteristic of the present system is that it not only assesses the average emotionality when stimuli are presented, but also shows the trend of change in emotionality over time.
\end{abstract}

In order to measure human emotionality that indicates the intensity of emotion, research has mainly focused on measuring diverse aspects of human behavior, which include behavioral changes, subjective assessment, and physiological responses. In the beginning, psychologists tried to understand psychological states of humans through the discrete state theory and argued that human emotion can be classified into a specified number of categories (Ekman, 1972; Izard, 1971; Tomkins, 1984). Ekman (1972) defined the categories as the six basic emotions, such as happiness, surprise, fear, rage, hatred, and sadness. According to a dimensional model, the psychological state is continuous and can be placed in a bipolar multidimensional space. This study claimed that the psychological state was composed of either three dimensions or fewer than three dimensions. For example, Schlosberg (1952) asserted that emotion was composed of two bipolar dimensions and could be placed in two-dimensional space as a circular shape.

Russell (1980) also suggested a spatial model based on a statistical study of 28 words that represent psychological states. He suggested that each emotion could be positioned in a two-dimensional space, including the axes of pleasantness-unpleasantness and arousal-relaxation, in a circular shape. The two-dimensional structure has been consistently found in studies that investigated the dimensions of psychological and emotional states via emotional structure or facial expressions of different cultural backgrounds (Osgood, May, \& Miron, 1975; Russell, Lewicka, $\&$ Niit, 1989). Therefore, the two dimensional structure is considered a fairly stable, general structure for graphically presenting human emotionality.

In order to evaluate human emotionality, both subjective assessment and analysis of physiological signals have been employed. Subjective assessment is a method that measures emotions retrospectively, using questionnaires. The first step in subjective assessment using questionnaires is to set the goal of the investigation. The second step involves assembling adjectives that represent a specific area of interest. The third step involves selecting the proper adjectives from the existing pool, and the final step entails evaluating emotions for the presented stimulus.

Subjective assessment, however, is limited in the way that it is completed after the presentation of a stimulus (or stimuli). Therefore, evaluation is done retrospectively from memory of the stimulus and not from the stimulus itself. When the magnitude of the stimulus is low or the experimental time is rather long, the real emotions felt at the point to be measured may not be expressed properly.

S.-C. Chung, scchung@kku.ac.kr 
Wierwille and Eggemeier (1993) pointed out that as the experimental time grew or the contents of the experiment became more complex, subjective evaluation produced serious problems in the measurement of the subjective workload. To overcome this problem, Tattersall and Foord (1996) measured the subjective workload on a 5-point scale that had five points of self-evaluated workload, such as excessive, high, comfortable, relaxed, and under utilized, using the Instantaneous Self Assessment (ISA) in real time.

Many studies have been conducted on measuring electrical/physiological signals as a method for objective evaluation of emotion (Davidson \& Fox, 1982; Ekman, Levenson, \& Friesen, 1983; Elliott, 1974; Elul, 1972; Fox, 1991; Hinrichs \& Machleidt, 1992; Kostyunina \& Kulikov, 1996; Levenson, Ekman, \& Friesen, 1990; Rosenberg \& Ekman, 1994). Heart-rate variability (HRV), galvanic skin response (GSR), skin temperature, and electroencephalogram (EEG) are known to be physiological signals reflecting different emotional properties. In particular, GSR sensitivity, which is used to cover not only phasic electrodermal responses but also electrodermal phenomena in general, including tonic electrodermal activity (EDA) that is measured in various parameters (i.e., mean amplitude, frequency of peaks, deviation from baseline, trends etc.), reflects levels of arousal (Kak, 1981) and is clearly higher during the portrayal of negative emotions than during that of positive emotions (Levenson et al., 1990). Some research reports that GSR also reflects levels of orienting response, awareness, and attention (Andreassi, 1995). Lee and Olness (1996) observed GSR to pleasant and exciting images, and Glaros (1996) observed GSR to horror movies. Patterns of GSR varied according to the type of emotional stimulation, but the research produced consistent results showing that physiological excitement increases along with the intensity-measured in the average amplitude - of GSR (Min, Chung, Min, \& Sakamoto, 2004; Min et al., 2002).

Objective evaluation of emotion using physiological signals is able to show trends of emotional change over time as well as average emotional changes for the whole duration of the presentation of the stimuli. In contrast, questionnaires are limited in observing trends of emotional change over time. Therefore, results from the subjective assessment cannot be compared with physiological signals because of differences in the time of measurement. That is, subjective evaluation can neither be compared with the physiological signals on a one-to-one basis, nor can it measure the emotion felt at the moment of presentation of the stimulus.

The present study developed a real-time subjective emotionality assessment (RTSEA) system to overcome limitations in traditional methods of the subjective assessment using questionnaires. Russell's model (1980), which assumes two dimensions of pleasantness-unpleasantness and arousal-relaxation, was used for the present study, because the two dimensions (valence and arousal) are considered to be stable for selecting emotional stimuli-even if some researchers (e.g., Lang, Bradley, \& Cuthbert, 1997) propose a tridimensional theory of emotion. The present study developed the system that allowed subjects to express their emotions on a digitized input board having two axes - one for pleasantness-unpleasantness and the other for arousal-relaxation. Subjects placed the penmouse at the exact position of the input board the moment they experienced the aforesaid emotion. The results were processed and displayed on a monitor in real time.

The present study measured GSR and the RTSEA at the same time during the presentation of a picture that aroused positive emotionality and another one that aroused negative emotionality. Following the experiment, the subjects were asked to complete the subjective assessment using a questionnaire. This study compared the results of the RTSEA with the subjective assessment on a questionnaire and GSR responses.

\section{THE RTSEA SYSTEM}

The RTSEA system using digitizer is composed of an emotionality input part, a connection between the digital tablet and the host computer (RS232C), and an emotionality evaluation and display parts.

When the program starts, the experimenter sets the range of the digital tablet and then sets the measurement parameters. That is, he or she determines the point scale to be used for subject evaluation (5- or 7-point scale or continuous scale) and the size of the data sampling (10 points/ $\mathrm{sec})$. The experimenter then enters the measurement time, subject-related and experiment-related information, and so on. When preparations for measurement are completed, the subject is given the digital tablet, and real-time subjective evaluation (via the digitizer) is conducted on emotionality stimulation. When measurement is completed, the results of the measurement and analysis are printed out or stored.

\section{Input}

The input part is a digital tablet (Wacom Intuos, Japan) the size of an A4 sheet of paper. It is designed in such a way that it can be marked with a digital stylus for input (area, $127 \times 99 \mathrm{~mm}$; resolution, $0.01 \mathrm{~mm}$; accuracy, $\pm 0.25 \mathrm{~mm}$; speed, 200 points/sec). As shown in Figure 1A, the digital tablet was designed so that subjects could express their emotionality easily and correctly.

Subjects were instructed to mark the digital tablet by placing the stylus on the two-dimensional input board with the pleasantness-unpleasantness dimension on the horizontal axis and the arousal-relaxation dimension on the vertical axis in real time. The sampling rate for data collection from the stylus was 10 points $/ \mathrm{sec}$. The bipolar 5-point scale $(-2,-1,0,1,2)$ was used for the input board in this study. Subjects were instructed that when the stimulus was very pleasant, point +2 should be marked on the horizontal axis and when the stimulus was very unpleasant, point -2 should be marked on the horizontal axis. When the stimulus was very arousing, point +2 should be marked on the vertical axis and when the stimulus was very relaxing, point -2 should be marked on the vertical axis (see Figure 1A). The two-dimensional input board was composed of 25 separate areas on an $18 \mathrm{~mm}^{2}$ square grid. 

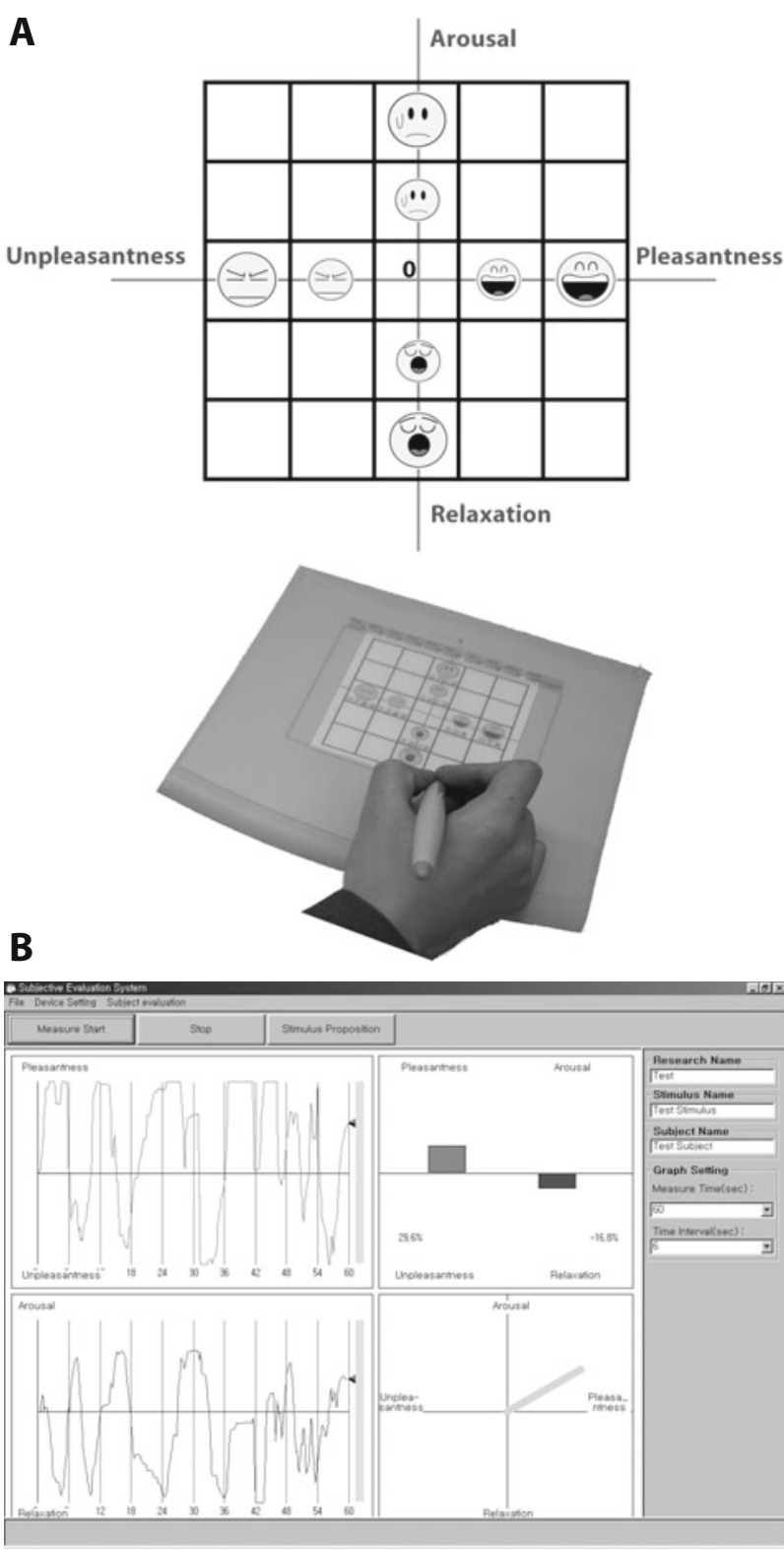

Figure 1. The RTSEA System. (A) The input board of the digital tablet equipped with stylus input device. (B) The part for the evaluation of human emotionality and display.

\section{Evaluation of Human Emotionality and Display}

As in Figure 1B, the results from both the one-dimensional and two-dimensional evaluations can be displayed in real time on the monitor. The upper left side displays the time series for pleasantness-unpleasantness, and the lower left side displays the time series for arousal-relaxation ( $x$-axis represents the flow of time, $y$-axis represents the value of human emotionality). The lower right side displays the change in emotion in real time on the two-dimensional space as a line. The upper right area displays the cumulative results of emotion for each dimension during the time of the presentation of the stimuli. The cumulative evaluation of emotion for each dimension was derived by integrat- ing the values in the dimension of emotion with time. For the present system, a technique was developed to measure the change in emotion as well as the cumulative degree of change in emotion in real time.

\section{REAL-TIME SUBJECTIVE EMOTIONALITY EVALUATION ON POSITIVE AND NEGATIVE VISUAL STIMULI}

\section{Subjects and Procedure}

The subjects for this study included 28 college graduates; 14 males within the age range of $25.3 \pm 3.5$ years and 14 females between the ages of $24.2 \pm 3.1$ years. The subjects participated in the experiment after receiving instructions about the contents of the experiment in detail and by practicing the response of emotionality on the digital tablet for $30 \mathrm{~min}$. They were told to not move, to make themselves comfortable, to concentrate on the stimulus, and to express their emotions as accurately as possible. They all reported that they were not under any kind of stress and did not have a mental workload or problems in expressing their emotions on the digital tablet.

For the experiment, two pictures from the international affective picture system (IAPS) that had been previously verified to evoke positive and negative emotions were used as the visual stimuli (Lang et al., 1997). The visual stimuli were presented at random for $300 \mathrm{sec}$ each. The presentation of the stimulus was projected on a screen that was $1.5 \times 1.8 \mathrm{~m}$ (Sanyo, PLC-5600N, Japan) in size. During the period that the subject evaluated his or her emotions for the stimulus on the digital tablet, GSR was measured. BIOPAC MP100 was used to measure the average amplitude of GSR from the index and middle fingers on the left hand. The sampling rate of the physiological data was $256 \mathrm{~Hz}$. At the end of the experiment, subjective evaluation lasted for 1 min while the subjects filled out a questionnaire.

\section{Subjective Assessment Using the Questionnaire}

For subjective assessment using the questionnaire, 12 adjectives that are considered to evaluate the degree of pleasantness and arousal were used on a bipolar 5-point scale. The six pairs of adjectives selected for measuring the pleasantness dimension were despairing-hopeful, boredpeaceful, unpleasant-pleasant, depressed-contented, miserable-happy, and dissatisfied-satisfied (Cronbach's $\alpha=.88$ ). Another six pairs of adjectives for measuring the arousal dimension were numb-nervous, languid-excited, dull-animated, sleepy-wide awake, calm-restless, and drowsy-not drowsy at all (Cronbach's $\alpha=.79$ ). The averaged scores of the six pairs of adjectives for each dimension of pleasantness and arousal were calculated.

\section{RESULTS}

\section{Results of the RTSEA}

The results of the real-time subjective evaluation obtained from the positive and negative visual stimuli that were presented continuously for $300 \mathrm{sec}$ each are shown 
in Figures 2 and 3, respectively (averaged for 28 subjects). Data were collected at 10 points/sec, with the presented data averaged for each 5-sec interval. The results are shown in such a way that the two-dimensional axes of pleasantness-unpleasantness and arousal-relaxation were separated and displayed as one dimension.

As shown in Figures 2A and 2B, a positive visual stimulus aroused pleasant and relaxed subjective emotionality in general, and the averages on emotionality that were aroused during the stimulation were $0.63 \pm 0.31$ and $-0.99 \pm 0.45$, respectively. A negative visual stimulus aroused unpleasantness and arousal emotionality in general, and the average emotionality aroused during the stimulation was $-1.54 \pm 0.41$ and $0.86 \pm 0.39$, respectively (Figures $3 \mathrm{~A}$ and $3 \mathrm{~B}$ ). In addition, the difference in the change of emotionality over time was observed for the two visual stimuli. Emotionality caused by the positive visual stimulus decreased significantly over time, in comparison with that caused by the negative visual one.
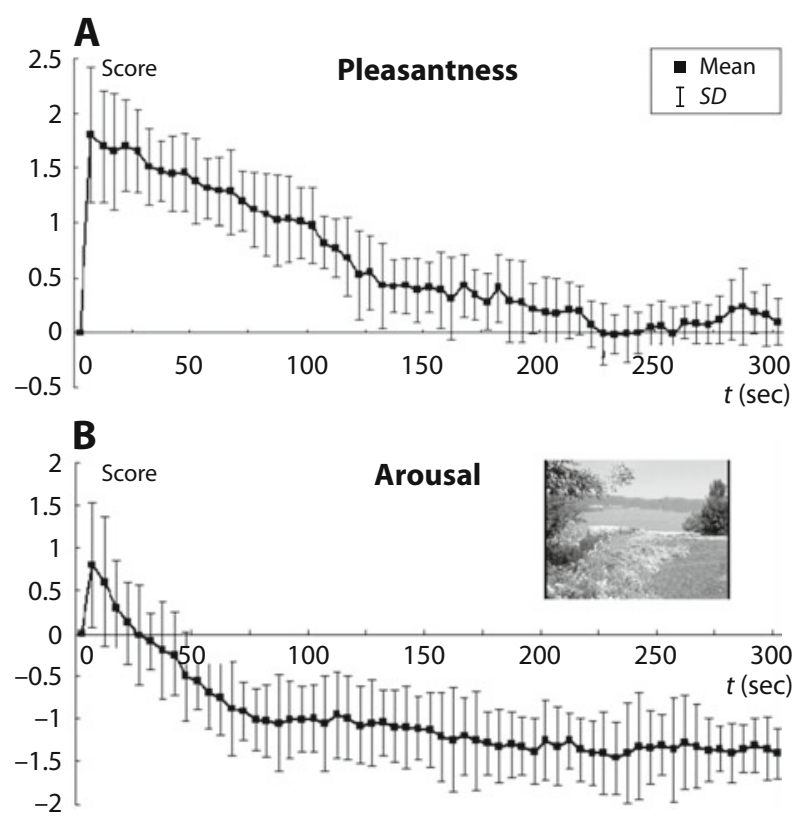

\section{C}

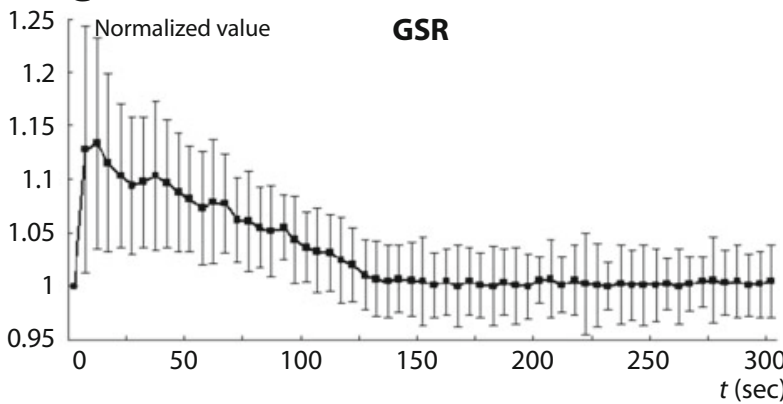

Figure 2. Results of the RTSEA and GSR on positive visual stimuli. (A) Changes in the level of pleasantness over time. (B) Changes in the level of arousal over time. (C) Changes in GSR over time.

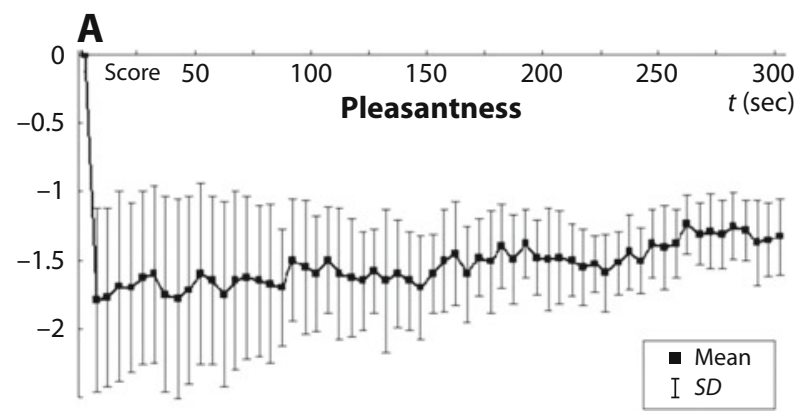

B
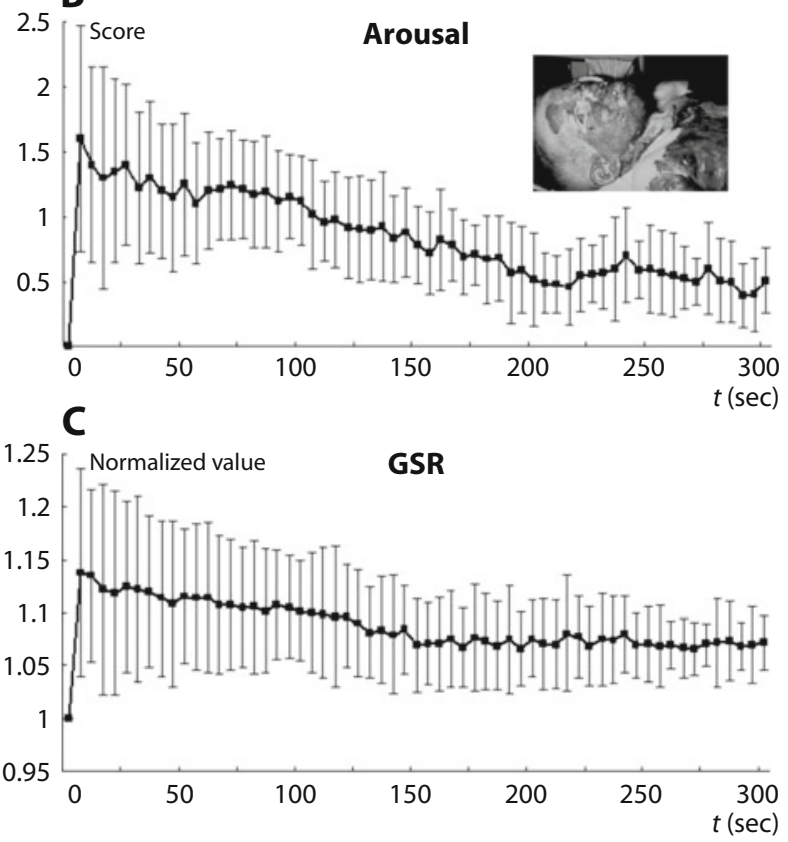

Figure 3. Results of the RTSEA and GSR on negative visual stimuli. (A) Changes in the level of pleasantness over time. (B) Changes in the level of arousal over time. (C) Changes in GSR over time.

\section{Comparison of Results Between GSR and the RTSEA}

GSRs were collected at 256 points/sec, and the present data were the average of each 5-sec interval (Figures $2 \mathrm{C}$ and $3 \mathrm{C}$ ). In addition, the data were normalized on the basis of $t=0$, and the average was calculated from all 28 subjects. GSRs to both positive and negative visual stimuli increased abruptly at the beginning and gradually decreased over time. However, there was a difference between GSR patterns of the positive and negative stimuli. For the positive visual stimulus, the GSR response decreased rapidly over time and reached baseline after $130 \mathrm{sec}$. For the negative visual stimulus, however, the GSR response decreased gradually over time and stayed at a constant value after about $130 \mathrm{sec}$. Similar to the results of the RTSEA, GSR responses to a positive visual stimulus decreased more rapidly over time than those to a negative visual stimulus. The results suggest that a negative visual stimulus arouses physiological excitement longer than a positive visual stimulus. Table 1 shows the 
Table 1

Correlation Between Changes in RTSEA and GSR Responses Aroused by Positive and Negative Visual Stimuli

\begin{tabular}{ccrc}
\hline & & \multicolumn{2}{c}{ GSR } \\
\cline { 3 - 4 } RTSEA & & Positive & Negative \\
\hline Positive & Pleasure & $.966^{* *}$ & $.904^{* *}$ \\
& Arousal & $.875^{* *}$ & $.637^{* *}$ \\
\multirow{2}{*}{ Negative } & Pleasure & $-.494^{* *}$ & $-.784^{* *}$ \\
& Arousal & $.879^{* *}$ & $.942^{* *}$ \\
\hline
\end{tabular}

${ }^{* *} p<.01$.

correlation coefficients (Pearson $r$ ) between changes in subjective assessment caused by visual stimuli and GSRs. Because both changes in the RTSEA and GSR response for each visual stimulus increased at the beginning of the stimulation and decreased over time, the correlation between the two appeared extremely high, as shown in Table 1. However, when comparing this correlation with that of the change in the RTSEA and GSR for each emotional stimulus, the following differences were found. When a positive visual stimulus was presented, the correlation between change in the subjective emotionality of pleasantness and $\mathrm{GSR}_{\text {positive }}(.966, p<.01)$ was higher than that with $\operatorname{GSR}_{\text {negative }}(.904, p<.01)$, and, similarly, change in the subjective emotionality of arousal was more closely correlated with $\operatorname{GSR}_{\text {positive }}(.875, p<.01)$ than with $\mathrm{GSR}_{\text {negative }}(.637, p<.01)$. When a negative visual stimulus was presented, the correlation between change in the subjective emotionality of pleasantness and GSR $\mathrm{Gegative}$ $(-.784, p<.01)$ was higher than that with $\mathrm{GSR}_{\text {positive }}$ $(-.494, p<.01)$, and, similarly, change in the subjective emotionality of arousal was more closely correlated with $\operatorname{GSR}_{\text {negative }}(.942, p<.01)$ than with $\operatorname{GSR}_{\text {positive }}(.879, p<$ .01 ). (Here, GSR positive $_{\text {and }} \mathrm{GSR}_{\text {negative }}$ are the results of GSR measured with positive and negative visual stimuli, respectively.) These results suggest that the RTSEA system is very effective for observing the change in subjective emotionality over time.

\section{Comparison of Results Between Subjective Evaluation Using the Questionnaire and the RTSEA}

The cumulative values of emotion for each axis of pleasantness and arousal for the two subjective evaluations were compared. For each emotional dimension and stimulus, mean gain scores were calculated for the RTSEA responses and the subjective responses on questionnaire, and one sample $t$ test was performed on the gain scores to compare the two response sets obtained from the two methods. As shown in Figure 4, for the positive visual stimulus, no significant difference was found between the two on pleasantness (RTSEA $=0.63 \pm 0.31$; questionnaire $=0.98 \pm 0.52)[t(27)=2.01$, n.s. $]$ and on arousal $(\operatorname{RTSEA}=-0.99 \pm 0.45 ;$ questionnaire $=-0.84 \pm 0.63)$ $[t(27)=-1.24$, n.s. $]$. For the negative stimulus, the value of pleasantness $(\mathrm{RTSEA}=-1.54 \pm 0.41$; questionnaire $=$ $-1.47 \pm 0.47)[t(27)=-1.62$, n.s. $]$ and arousal (RTSEA $=$ $0.86 \pm 0.39$; questionnaire $=0.85 \pm 0.67)[t(27)=-0.21$, n.s.] were similar to each other for the two methods, and as for the case of positive stimulus, there was no significant difference between the two methods.

The results showed that there was no difference between the results of both the methods for subjective evaluation using the questionnaire after the presentation of the stimulus and the RTSEA method. From these results, we concluded that the subjective evaluation using a questionnaire could represent the average level of emotion for the entire time of the presentation of the stimulus, and the RTSEA could represent not only the average level of emotion but also the trend of change in emotionality over time.

\section{DISCUSSION}

The present study investigated the feasibility and effectiveness of the RTSEA. The RTSEA is composed of two parts: the emotionality input and the evaluation and display parts. The input part allows subjects to express their emotionality on an A4-size digital tablet using a stylus. The input board of the RTSEA has two-dimensional axes - the pleasantness-unpleasantness and the arousal-relaxation axes. The evaluation of the input is done in real time. The
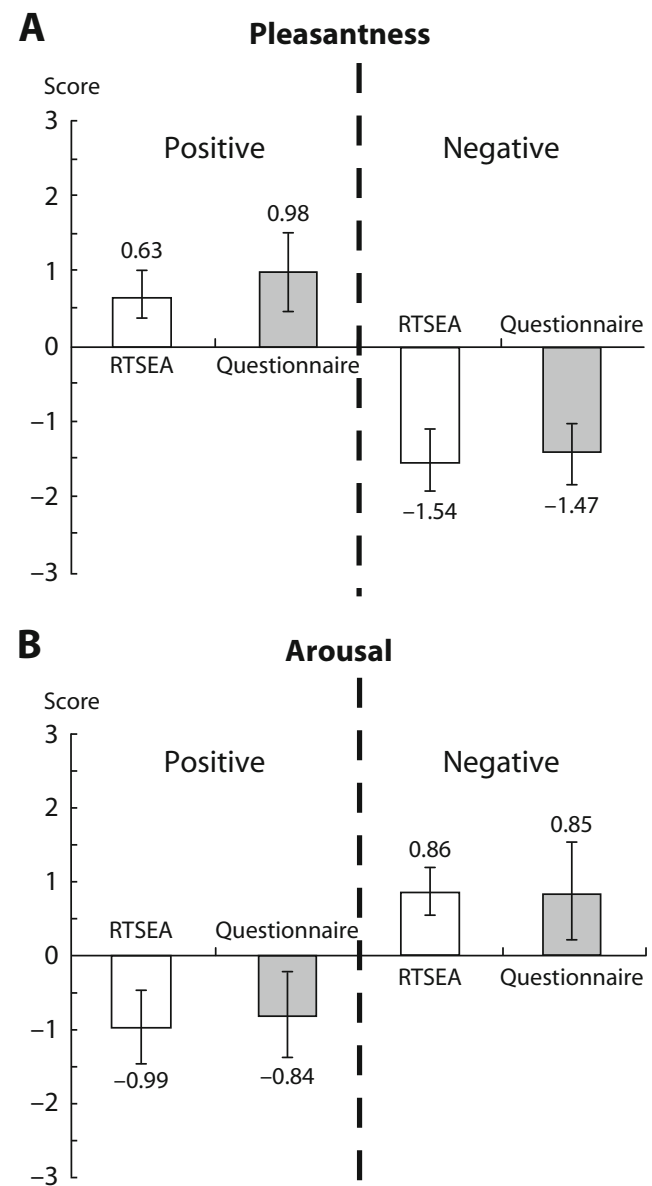

Figure 4. Comparison of the results of the RTSEA and subjective evaluation using the questionnaire. (A) Average value of pleasantness for positive and negative stimuli. (B) Average value of arousal for positive and negative stimuli. 
evaluation and display part interfaces with the input part and analyzes and displays the results for each dimension separately, in real time. It also displays the averaged emotionality for the elapsing time of the presentation in twodimensional space. The advantage of the present system is that it is able to evaluate human emotionality in real time and makes a comparison of physiological signals on a one-to-one basis, as well as averages values for human emotionality. The system is also able to create a file that can be used as part of a database.

For the present study, the subjects evaluated their emotionality for the presented positive and negative stimuli to verify the feasibility and effectiveness of the RTSEA. In addition, this study attempted to determine if change in subjective emotionality over time observed using the RTSEA system was meaningful. For this purpose, both the RTSEA and the measurement of GSR - a physiological signal closely related to physiological excitementwere performed and their results were compared. After the termination of the stimuli, the subjective assessment using a questionnaire was performed for comparing with the results of the RTSEA. The results indicate that the traditional subjective questionnaire reflects only the averaged emotionality, but that the RTSEA can represent not only the average level of emotion, but also the trend of change in emotionality over time. Therefore, the RTSEA system can be applied to those industries that require information on human emotionality that changes moment by moment. For example, the RTSEA can be used for gaining insight into the emotionality that is evoked of customers while watching TV commercials. If emotionality that changes with time can be measured and evaluated accurately by using the RTSEA system, then it will present a lot of information for designing a product that fits human emotion.

The results of the experiment using the RTSEA system showed that a positive visual stimulus generally showed pleasant and relaxed emotionality, and the negative visual stimulus showed unpleasant and arousal emotionality. Difference in the change of emotionality over time was observed between the two visual stimuli. That is, emotionality caused by the positive visual stimulus decreased significantly over time in comparison with that caused by the negative visual stimulus.

GSR response was closely related to physiological excitement, as well as to change in emotionality over time. In comparison with the negative visual stimulus, when the positive visual stimulus was presented, GSR response also decreased rapidly over time. According to the comparison of correlation coefficients, change in subjective emotionality caused by the positive visual stimulus was related more closely to GSR reaction caused by stimulation of the positive visual stimulus, and changes in subjective emotionality caused by the negative visual stimulus was related more closely to GSR reaction caused by stimulation with the negative visual stimulus. These results indicate that the RTSEA system is a viable method for objectively representing changes in subjective emotionality over time.

Traditional subjective evaluation using the questionnaire produced similar values to the averaged value of the RTSEA. Comparisons in terms of the numerical values of emotionality from the real-time and traditional retrospective methods of subjective emotionality evaluation may need more thorough discussion in the future, but we argue in the present study that subjective evaluation using the questionnaire reflected averaged emotionality for the entire duration of the presentation of the stimulus. Hence, the RTSEA could be used widely for measuring human emotionality since it is able to measure changes in the level of emotion in real time as well as in the averaged value of the subjective evaluation for the entire time of the presentation of the stimulus.

The greatest concern was given to the degree of subjective workload and the degree of accuracy in expressing emotionality. Subjects for the present experiment reported that they didn't have any type of workload or feel any stress, though any type of workload or stress could be an interfering factor in emotionality evaluation. Continuous development or improvement of the system is necessary for subjects to be able to express their emotionality more easily. Also, the present study used the fixed two-dimensional space that has axes of pleasantness-unpleasantness and arousal-relaxation for the positive and negative stimulus, but a different study with different experimental goals and evaluation may use other diversified dimensions different from the two dimensions used in the present study.

\section{AUTHOR NOTE}

This work was supported by grant R01-2004-000-10593-0 from the basic research program of the Korea Science and Engineering Foundation. Address correspondence to S.-C. Chung, Department of Biomedical Engineering, Konkuk University, 322 Danwall-dong, Chungju, Chungbuk, 380-701, South Korea (e-mail: scchung@kku.ac.kr).

\section{REFERENCES}

Andreassi, J. (1995). Psychophysiology: Human behavior and physiological response. Hillsdale, NJ: Erlbaum.

DAVIDSON, R. J., \& Fox, N. A. (1982). Asymmetrical brain activity discriminates between positive and negative stimuli in human infants. Science, 218, 1235-1237.

EKMAN, P. (1972). Universal and cultural differences in facial expressions of emotion. In J. K. Cole (Ed.), Nebraska symposium on motivation (pp. 207-283). Lincoln: University of Nebraska Press.

Ekman, P., Levenson, R. W., \& Friesen, W. V. (1983). Autonomic nervous system activity distinguishes among emotions. Science, 22, 1208-1210

Elliott, R. (1974). The motivational significance of heart rate. In P. A. Obrist, A. H. Black, J. Brener, \& L. V. DiCara (Eds.), Cardiovascular psychophysiology: Current issues in response mechanisms, biofeedback, and methodology (pp. 505-537). Chicago: Aldine.

ELUL, R. (1972). The genesis of the EEG. International Review of Neurobiology, 15, 227-272.

Fox, N. A. (1991). If it's not left, it's right: Electroencephalograph asymmetry and the development of emotion. American Psychologist, $\mathbf{4 6}$, 863-872.

Glaros, A. G. (1996). Awareness of physiological responding under stress and nonstress conditions in temporomandibular disorders. Biofeedback \& Self-Regulation, 21, 261-272.

HinRICHS, H., \& MACHLEIDT, W. (1992). Basic emotions reflected in EEG-coherences. International Journal of Psychophysiology, 13, 225-232.

IZARD, C. E. (1971). The face of emotion. New York: Appleton-CenturyCrofts.

KAK, A. V. (1981). Stress: An analysis of physiological assessment devices. In G. Salvendy \& M. J. Smith (Eds.), Machine pacing and occupational stress. London: Taylor \& Francis.

Kostyunina, M. B., \& Kulikov, M. A. (1996). The frequency charac- 
teristics of EEG spectra in the emotions. Neuroscience \& Behavioral Physiology, 26, 340-343.

LANG, P. J., Bradley, M. M., \& Cuthbert, B. N. (1997). International affective picture system (IAPS): Technical manual and affective ratings. Gainesville, FL: NIMH Center for the Study of Emotion and Attention.

LEe, L. H., \& OlNEss, K. N. (1996). Effects of self-induced mental imagery on autonomic reactivity in children. Journal of Developmental \& Behavioral Pediatrics, 17, 323-327.

Levenson, R. W., Ekman, P., \& Friesen, W. V. (1990). Voluntary facial action generates emotion-specific autonomic nervous system activity. Psychophysiology, 27, 363-384.

Min, B. C., Chung, S. C., Min, Y. K., \& Sakamoto, K. (2004). Psychological evaluation of simulator sickness evoked by a graphic simulator. Applied Ergonomics, 35, 549-556.

Min, B. C., Chung, S. C., Park, S. J., Kim, C. J., Sim, M.-K., \& SaKaмото, K. (2002). Autonomic responses of young passengers contingent to the speed and driving mode of a vehicle. International Journal of Industrial Ergonomics, 29, 187-198.

Osgood, C. E., MaY, W. H., \& Miron, M. S. (1975). Cross-cultural universals of affective meaning. Urbana: University of Illinois Press.
Rosenberg, E. L., \& Ekman, P. (1994). Coherence between expressive and experiential systems in emotion. Cognition \& Emotion, $\mathbf{8}$, 201-229.

Russell, J. A. (1980). A circumplex model of affect. Journal of Personality \& Social Psychology, 39, 1161-1178.

Russell, J. A., LeWICKA, M., \& NitT, T. (1989). A cross-cultural study of a circumplex model of affect. Journal of Personality \& Social Psychology, 57, 848-856.

SCHLOSBERG, H. (1952). The description of facial expressions in terms of two dimensions. Journal of Experimental Psychology, 44, 229-237.

TATTERSAll, A. J., \& Foord, P. S. (1996). An experimental evaluation of instantaneous self-assessment as a measure of workload. Ergonomics, 39, 740-748.

TomkIns, S. S. (1984). Affect theory. In K. R. Scherer \& P. Ekman (Eds.), Approaches to emotion (pp. 163-195). Hillsdale, NJ: Erlbaum.

Wierwille, W. W., \& EgGEMeier, F. T. (1993). Recommendations for mental workload measurement in a test and evaluation environment. Human Factors, 35, 263-281.

(Manuscript received November 6, 2004; revision accepted for publication December 13, 2005.) 